\title{
Management of inguinal hernias in premature infants- pre or post discharge- what is best?
}

\begin{abstract}
Incidence of inguinal hernias in premature infants is reported to be as high as 30\%, making inguinal hernia repair one of the most common surgeries performed in children. Frequency of inguinal hernia is inversely related to birthweight with rates highest in the very low birth weight infants. Due to multiple comorbidities, most pediatric surgeon weight to operate on premature infants until they have reached $1800-2000 \mathrm{~g}$. The purpose of this retrospective study is to determine optimal time period for repair of inguinal hernias in premature infants and to establish the use of regional anesthesia as standard of care. In a retrospective chart review of premature infants undergoing inguinal hernia repair from 1997 to 2016, 134 cases were reviewed. Anesthesia complications were limited to $0.07 \%$ in the caudal group and none in the spinal anesthesia group. Hernia recurrence rate in this study was minimal at $0.01 \%$. Inguinal hernias in premature infants should be repaired as early as they are healthy enough to be transferred out of the intensive care unit, taking adequate oral nutrition and do not have further supplemental oxygen requirements. Regional anesthesia should be anesthesia of choice in premature infants.
\end{abstract}

Keywords: inguinal hernia, premature infants, regional anesthesia, timing of surgery

Volume 7 Issue I - 2017

Farda Qayyum,' Domingo Alvear²

'Department of General Surgery, Pinnacle Health System, USA

${ }^{2}$ Department of Pediatric Surgery, Pinnacle Health System, USA

Correspondence: Farda Qayyum, General Surgery Residency Program, Pinnacle Health System. 30I Chestnut St, \#I I03, Harrisburg, PA 17|0I, USA, Tel 7|4-232-2637, Fax 7|7-23|8756, Email fqayyum@pinnaclehealth.org

Received: August 29, 2016 | Published: July 21, 2017

\section{Introduction}

Inguinal hernias are one of the most commonly performed surgical procedure in children. ${ }^{1}$ The incidence of inguinal hernias is up to $5 \%$ with an even higher incidence in premature infants. Incidence of inguinal hernias in premature infants is reported to be as high as $30 \%$ with male predominance and rates 3-4 times higher than in girls. ${ }^{2-4}$ Frequency of inguinal hernia is inversely related to birthweight with rates highest in the $500-1000 \mathrm{~g}$ population. ${ }^{5}$ This weight group is almost three times more likely to require emergent surgery due to incarceration. The risk of incarceration is doubled in premature neonates with inguinal hernia when repair is delayed beyond 40 weeks post-conceptual age. ${ }^{2}$ Approximately half of these low birthweight infants are found to have bilateral hernias.

Due to multiple comorbidities, immediate hernia repair is not frequently a safe, viable option. Incarceration reported in as many as $25 \%$ of infants awaiting elective repair. Only $63 \%$ of pediatric surgeons report routinely repairing inguinal hernia before infants are discharge from the hospital. Delaying repair increases the likelihood of a recurrence to $31 \%$, compared to only $5 \%$ if repaired during the initial hospitalization. Typically inguinal hernia repairs are done under general anesthesia which has a high risk of post-operative complications, especially in the premature infant. Complication rate is as high as $12 \%$ and includes apnea, bradycardia, long term behavioral and learning disabilities. Using general anesthesia has been associated with prolonged length of stay as well (Figure 1). ${ }^{6}$

There is a lack of general consensus among the surgical community regarding the timing of hernia repair. Many surgeons repair inguinal hernias before discharge from the neonatal intensive care until once the infants reach 1800 to $2000 \mathrm{~g} .{ }^{7}$ Due to a high incidence of bilateral inguinal hernias, contralateral exploration is often performed but remains a point of debate.

The purpose of this retrospective study is to determine optimal time period for repair of inguinal hernias in premature infants and to establish the use of regional anesthesia as standard of care.

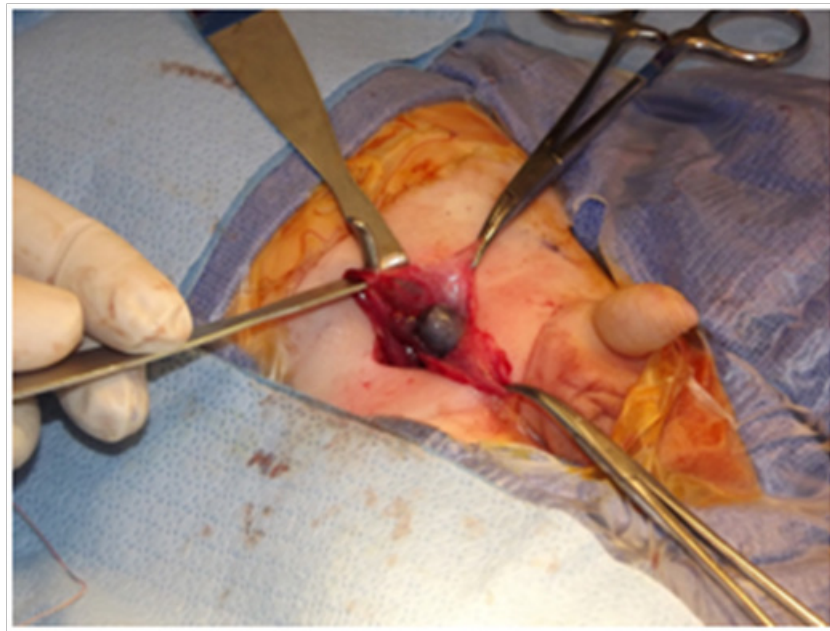

Figure I Incarcerated inguinal hernia with ischemic testicle.

\section{Materials and methods}

This study is a retrospective chart review of premature infants undergoing inguinal hernia repair at our institution. Premature infants undergoing inguinal hernia repairs from 1997 to 2016 were included in the study. Our institutional practice is to repair inguinal hernias before the patients were discharged from the neonatal intensive care unit, regardless of birth and current weight. Criteria for surgical intervention included no supplemental oxygen requirement or oral feeds.

The open technique is employed and routine contralateral open groin exploration is performed for all patients. All inguinal hernias are performed under spinal or caudal anesthesia to minimize the risk of apnea and bradycardia. Intraoperative monitoring, supplemental local anesthetic and temperature maintenance were provided (Figure $2 \& 3$ ). 

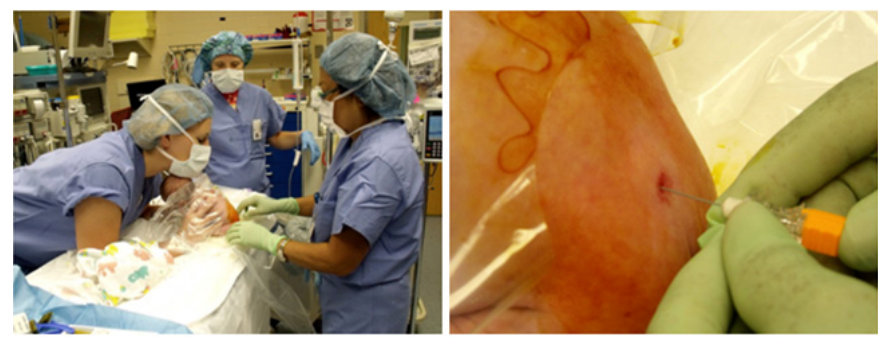

Figure 2 Spinal anesthesia.

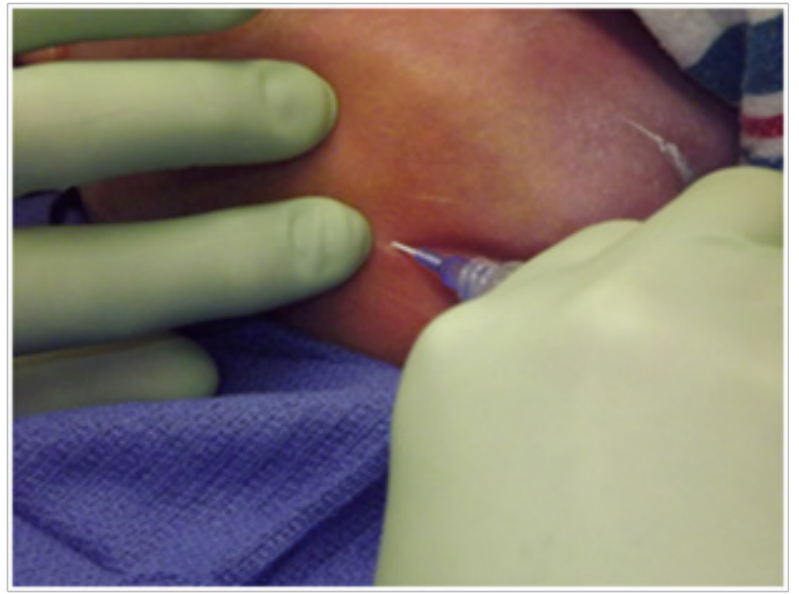

Figure 3 Caudal anesthesia.

\section{Results and discussion}

During the 20 year period, 134 premature infants underwent inguinal hernia repair at our institution. No patients were included for four of the years due to incomplete medical records. A total of three patients had incarcerated hernias at the time of presentation. Of the 134 hernia repairs, 54 were done under caudal anesthesia and 80 were done under spinal anesthesia. Average birth weight for the caudal anesthesia group was $1370 \mathrm{~g}$ and $1200 \mathrm{~g}$ for the spinal group. At the time of surgery the infants weighed in a range of 2000-4000 $\mathrm{g}$ and $1500-3500 \mathrm{~g}$ for the two groups respectively. Age at the time surgery ranged from 7 to 105 days for the caudal group and 20-96 days for the spinal group (Table 1).

Table I Patient demographics of 134 premature infants undergoing inguinal hernia repair

\begin{tabular}{lll}
\hline & Caudal & Spinal \\
\hline Number of Cases & 54 & 80 \\
Avg. Birth Wt. (g) & 1370 & 1200 \\
Wt. at time of Surgery $(g)$ & $2700(2000-4000)$ & $2400(1500-3500)$ \\
Age at Surgery (d) & $57(7-105)$ & $52(20-96)$ \\
\hline
\end{tabular}

Post-operative length of stay raged from 1 to 19 days in the caudal group and 1-45 days in the spinal group. Anesthesia complication was limited to $0.07 \%$ in the caudal group. Two patients in this group required conversion to general anesthesia due to apnea. Two patients required supplementation with sedation which was considered a complication. There were no anesthesia related complications in the spinal group. No clinically significant post-operative apneic or bradycardic episodes occurred. A $0.01 \%$ recurrence rate was noted in each of the groups. No wound infections occurred in either group (Table 2).
Table 2 Post-operative length of stay, incidence of hernia recurrence and anesthesia related complications including apnea, bradycardia, need for conversion to general anesthesia

\begin{tabular}{lll}
\hline & Caudal & Spinal \\
\hline Post-op LOS & $10(19)$ & $7(1-5)$ \\
Recurrence & 0.01 & 0.01 \\
Anesthesia Complications & 0.07 & None \\
\hline
\end{tabular}

Based on the above results we concluded that early repair is the best strategy in order to minimize incarceration. Many pediatric surgeons wait to perform surgery until the infants weight at least $1800 \mathrm{~g}$. In this study however, hernia repair is performed once the premature infants are healthy enough to be transferred out of the intensive care unit, taking adequate oral nutrition and do not have further supplemental oxygen requirements. Using these criteria, we have shown a minimal risk to operation regardless of patients' birth weight, weight at the time of operation and age. We have safely performed inguinal hernia repairs on infants weighing as low as $1500 \mathrm{~g}$ at the time of operation. We therefore recommend a shift from waiting for patients to meet minimal weight criteria to criteria used in this study. This will likely result in fewer incarcerations and fewer recurrences reducing the risks associated with emergent or repeat surgery in an already higher risk patient group.

Regional anesthesia, caudal or spinal is easy to perform using landmarks. It can be supplemented with local anesthetics as well as sedation if needed. Use of sedation with regional anesthesia should be minimized as it carries similar complications as general anesthesia. ${ }^{8,9}$ Regional anesthesia is designed for short procedures making it an adequate choice for inguinal hernia repairs. This type of anesthesia is much safer than general anesthesia which makes it a safe choice for premature infants allowing for early repair.

Use of general anesthesia in premature infants has been shown to increase their length of stay in the hospital. ${ }^{6}$ Using regional anesthesia we have been able to discharge patients home as early as post-operative day 1 . In this study, the post-operative length of stay ranged from 1-45 days with the majority of patients remained in the hospital due to social issues. As this study lacks a control group using general anesthesia, it is difficult to compare this parameter. We propose further prospective randomized trial using general anesthesia in the control group.

Contralateral exploration remains a controversial issue with many surgeons moving away from this practice. A detriment of bilateral groin exploration is longer time under anesthesia, however with regional anesthesia we feel that the increased time in the operating room is no clinically significant detrimental effects. With the continuing strides in advanced laparoscopic surgery, it is becoming more practical to perform a transinguinal evaluation of the contralateral side. This technique holds high sensitivity and specificity with minimal risks in the hands of a skilled surgeon. Therefor we recommend continued exploration of the contralateral side using whichever technique the surgeon is most comfortable with.

Our study lacks a comparison group matched for comorbidities that premature infants. A large number of patients were excluded from this analysis due to incomplete records which may have skewed the results. The pediatric surgery department at out institution is small without much variability in technique, making out results less applicable to the larger pediatric surgery practices. This data spans a 20 year period with a variable range of follow up. There may be hernia recurrences in patients from recent years which may change the data. Hence further follow up is needed in these patients. 


\section{Conclusion}

We conclude that inguinal hernias in premature infants should be repaired as early as they are healthy enough to be transferred out of the intensive care unit, taking adequate oral nutrition and do not have further supplemental oxygen requirements. No minimal weight requirements need to be met when using this criteria. Regional anesthesia is safer than general anesthesia, making it a safe choice for premature infants allowing for early hernia repair.

\section{Acknowledgments}

None.

\section{Conflicts of interest}

The authors declare no conflicts of interest.

\section{References}

1. Pini Prato A, Rossi V, Mosconi M, et al. Inguinal hernia in neonates and ex-preterm: complications, timing and need for routine contralateral exploration. Pediatr Surg Int. 2015;31(2):131-136.

2. Lautz TB, Raval MV, Reynolds M. Does timing matter? A national perspective on the risk of incarceration in premature neonates with inguinal hernia. J Pediatr. 2011;158(4):573-577.
3. Burgmeier C, Dreyhaupt J, Schier F. Gender-related differences of inguinal hernia and asymptomatic patent processus vaginalis in term and preterm infants. J Pediatr Surg. 2015;50(3):478-480.

4. Skoog SJ, Conlin MJ. Pediatric hernias and hydroceles. The urologist's perspective. Urol Clin North Am. 1995;22(1):119-130.

5. Harper RG, Garcia A, Sia C. Inguinal hernia: a common problem of premature infants weighing 1,000 grams or less at birth. Pediatrics. 1975;56(1):112-115.

6. Somri M, Gaitini L, Vaida S, et al. Postoperative outcome in highrisk infants undergoing herniorrhaphy:comparison between spinal and general anaesthesia. Anaesthesia. 1998;53(8):762-766.

7. Sulkowski JP, Cooper JN, Duggan EM, et al. Does timing of neonatal inguinal hernia repair affect outcomes? J Pediatr Surg. 2015;50(1):171176.

8. Craven PD, Badawi N, Henderson-Smart DJ, et al. Regional (spinal, epidural, caudal) versus general anaesthesia in preterm infants undergoing inguinal herniorrhaphy in early infancy. Cochrane Database Syst Rev. 2015;(3):CD003669.

9. Sanders RD, Hassell J, Davidson AJ, et al. Impact of anaesthetics and surgery on neurodevelopment: an update. Br J Anaesth. 2013;a110(Suppl 1):i53-i72. 